\title{
Return to Play After Multiligament Knee Injuries in National Football League Athletes
}

\author{
Neil K. Bakshi, MD, * Moin Khan, MD, Fred T. Finney, MD, Robby S. Sikka, MD, \\ and Asheesh Bedi, MD
}

Background: Return to play (RTP) of National Football League (NFL) athletes after isolated anterior cruciate ligament (ACL) tears has been reported. However, no studies have reported on RTP of NFL athletes after multiligament knee injuries.

Hypotheses: NFL athletes with multiligament knee injuries have lower RTP rates and longer recoveries than athletes with isolated ACL tears. Second, athletes with ACL and medial collateral ligament (MCL) injuries will have higher RTP rates and shorter time to RTP than athletes with an ACL tear and posterolateral corner involvement.

Study Design: Retrospective cohort study.

Level of Evidence: Level 4.

Methods: Publicly available NFL injury data were reviewed for all multiligament knee injuries incurred between 2000 and 2016 with RTP information. Athletes were excluded if RTP was limited for reasons unrelated to the injury. Extracted data included type of injury, RTP, time to RTP (days), number and percentage of games played, and performance.

Results: A total of 50 NFL athletes with multiligament knee injuries met inclusion and exclusion criteria. The overall RTP rate was $64.0 \%$. Athletes with ACL/MCL tears had an RTP rate of $70.8 \%$, whereas athletes with ACL and posterior collateral ligament/lateral collateral ligament (PCL/LCL) tears had an RTP rate of $55.6 \%(P=0.26)$. Mean time to RTP for all 50 athletes was $388.71 \pm 198.52$ days. The mean time to RTP for athletes with ACL/MCL injuries was 305.1 \pm 58.9 days, compared with $459.2 \pm 245.1$ days $(P=0.004)$ and 609.3 \pm 183.1 days $(P<0.0001)$ for those with combined ACL and PCL/LCL injuries and frank knee dislocations, respectively. Athletes with ACL/MCL injuries were more likely to return to prior performance level (43.5\%) than those with ACL and PCL/LCL injuries $(18.5 \%)(P<0.001)$.

Conclusion: The RTP rate for athletes with multiligament knee injuries is significantly less than the RTP rate for athletes with isolated ACL tears. In addition, athletes with ACL and MCL tears have a higher RTP rate, a significantly shorter time to RTP, and a greater likelihood of returning to prior performance than athletes with ACL and PCL/LCL tears.

Clinical Relevance: Multiligament knee injuries significantly affect the ability of a football player to return to sport. ACL and MCL tears are associated with better RTP prognosis compared with ACL and PCL/LCL tears.

Keywords: National Football League; return to play; anterior cruciate ligament; medial collateral ligament; posterior cruciate ligament; lateral collateral ligament

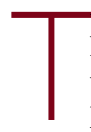
he physical demands of professional football result in a high rate of player injuries compared with many other professional sports. ${ }^{1,14}$ Often, players are unable to return to their previous level of performance after injury. ${ }^{14}$ Mai et $\mathrm{al}^{14}$ reported a $79.4 \%$ return-to-play (RTP) rate after orthopaedic intervention in National Football League (NFL) athletes. The epidemiology of common musculoskeletal injuries, such as anterior cruciate ligament (ACL) tears, has been well reported in the literature. Scranton et $a l^{17}$ reported the incidence of ACL injuries to be 0.7 per $1000 \mathrm{NFL}$ players based on an evaluation of 61 athletes. Bradley et $\mathrm{al}^{2}$ found an incidence of $53 \mathrm{ACL}$ injuries per year in the NFL. RTP after isolated ACL tears has 
also been well described in the literature, with reported RTP rates ranging from $63 \%$ to $92 \%$., ${ }^{5,14}$ The largest review on the subject was conducted by Mai et $\mathrm{al}^{14}$ in a case series of $165 \mathrm{NFL}$ athletes, which reported an RTP rate of $82.4 \%$ for professional football players who sustained ACL tears. Previous studies have reported that approximately two-thirds of NFL athletes are able to return to their prior level of statistical performance after undergoing an ACL reconstruction. ${ }^{5}$

While less common than isolated ACL tears, multiligament knee injuries are devastating injuries that can have serious consequences on the longevity of a player's career. These injuries are often associated with knee dislocations, tibial/ femoral fractures, and potentially significant neurovascular injury, including peroneal nerve or popliteal artery injury. ${ }^{7}$ Players with multiligament knee injuries have variable degrees of recovery depending on injury severity. ${ }^{7,9}$

The purpose of this study was to evaluate multiligament knee injuries in professional football players in the NFL to report on RTP and performance. Second, we aimed to identify prognostic characteristics of multiligament injuries that may allow for improved outcomes assessment. We hypothesized that NFL athletes with multiligament knee injuries would have lower RTP rates compared with those with isolated ACL tears. Furthermore, we hypothesized that athletes with concomitant ACL/medial collateral ligament (MCL) injuries would have higher RTP rates, shorter time to RTP, and improved return to prior performance rates compared with athletes with concomitant ACL and posterior collateral ligament/lateral collateral ligament (PCL/ LCL) involvement.

\section{METHODS}

Data were collected retrospectively for this study. Institutional review board exemption was granted for this research, as all the information is publicly available.

Patients with multiligament knee injuries were identified from publicly available NFL injury surveillance data. Multiple sources of publicly available information were searched and used, including NFL game summaries, play-by-play documents, weekly injury reports, press releases, newspaper archives, and player profiles. Players on an NFL roster were included in the study if they sustained a multiligament knee injury between the years 2000 and 2016. NFL athletes who sustained multiligament knee injuries prior to the year 2000 were excluded to maintain consistent management and surgical techniques. NFL athletes who were injured after the start of the 2015 NFL season and had not yet returned to play were excluded because of an inadequate amount of time for RTP. Athletes were also excluded if their RTP was prevented for reasons unrelated to the injury, such as suspension, unrelated injury, or personal matters, as indicated by NFL injury data.

Collected data included NFL position, injury, date of injury, RTP, time to RTP (months), number of games played, percentage of possible games played, position-specific performance measures (yards, touchdowns, sacks, interceptions, etc), and any accolades a player received (most valuable player [MVP], Super Bowl MVP, Pro Bowl, All-Pro, etc). Player demographics, injury information, and RTP data (date of injury, type of injury, RTP date, time to RTP, position) were obtained from the NFL injury surveillance database. Metrics such as number of games played, performance measures (yards, touchdowns, sacks, interceptions, etc), and any player accolades were obtained using publicly available sources, including NFL game summaries, injury reports, press releases, and online player profiles. Successful RTP was defined as participation in at least 1 play in a preseason or regular-season NFL football game after a multiligament knee injury. Return to play in another professional football league (NFL Europe, Arena Football League, Canadian Football League) was not considered RTP. Preseason game play was regarded as the date of RTP. Return to prior level of performance was defined as a player's postinjury statistical performance exceeding $80 \%$ of his preinjury statistical performance. A player's best statistical category (sacks, yards, touchdowns, interceptions, etc) was utilized to determine whether he attained his prior level of performance.

Statistical analysis was performed using SPSS software (version 11.5; IBM Corp). Quantitative variables, such as time to RTP, were statistically analyzed using unpaired, 2-tailed $t$ tests. Categorical variables were compared using chi-square statistics. Significance level was set at $P<0.05$. We performed multivariate logistic regression to ascertain the effects of age, position, and injury type.

\section{RESULTS}

\section{Demographics}

A total of 50 NFL athletes who met the study inclusion and exclusion criteria were found to have sustained multiligament knee injuries between the years 2000 and 2016. Forty-six percent $(23 / 50)$ of athletes had a combined ACL and MCL injury, while $44 \%$ (27/50) of athletes had multiligament knee injuries that involved the PCL or the $\mathrm{LCL} /$ posterolateral complex. In players with PCL/LCL involvement, 8 complete knee dislocations were identified. Thirty-two percent $(16 / 50)$ of athletes were primarily defensive players, while the remaining 68\% (34/50) were primarily offensive players. Sixteen percent $(8 / 50)$ of athletes played on the offensive/defensive lines, with the other $84 \%$ (42/50) of athletes playing skill positions not on the offensive/defensive lines (Table 1 ).

\section{Return to Play}

The overall RTP rate after multiligament knee injuries was 64.0\%. Athletes with ACL/MCL tears had an RTP rate of $70.8 \%$, and athletes with PCL/LCL involvement had an RTP rate of 55.6\% ( $P=0.26)$. Athletes with complete knee dislocations had a $50 \%$ RTP rate, which was less than that for those who did not sustain a knee dislocation (66.7\%), but not statistically significant $(P=0.61)$. Mean time to RTP for all 50 athletes was $388.71 \pm 198.52$ days. The mean time to RTP for athletes with ACL/MCL injuries was 305.1 \pm 58.9 days, compared with $459.2 \pm$ 
Table 1. Demographics of included athletes

\begin{tabular}{|l|c|c|c|}
\hline Injury & No. of Subjects & Age at Injury, y & Football Position \\
\hline ACL/MCL tears & 23 & $25.9 \pm 2.5$ & 6 WR/TE, 5 DB, 4 OL, 3 RB, 2 QB, 2 LB, 1 DL \\
\hline ACL tear + PCL/LCL involvement & 27 & $25.1 \pm 3.5$ & $6 \mathrm{RB}, 6 \mathrm{WR} / \mathrm{TE}, 5 \mathrm{DB}, 4 \mathrm{QB}, 3 \mathrm{LB}, 3 \mathrm{OL}$ \\
\hline
\end{tabular}

$\mathrm{ACL}$, anterior cruciate ligament; DB, defensive back; DL, defensive line; LB, linebacker; LCL, lateral collateral ligament; MCL, medial collateral ligament; OL, offensive line; PCL, posterior cruciate ligament; $Q B$, quarterback; RB, running back; TE, tight end; WR, wide receiver.

Table 2. Return-to-play results

\begin{tabular}{|c|c|c|c|c|}
\hline Injury & $\begin{array}{c}\text { RTP } \\
\text { Rate, \% }\end{array}$ & $\begin{array}{l}\text { Time to RTP, } \\
\text { days, } \\
\text { Mean } \pm \text { SD }\end{array}$ & $\begin{array}{c}\text { RTP } \\
\text { in Season After } \\
\text { Injury, \% }\end{array}$ & $\begin{array}{c}\begin{array}{c}\text { Return to } \\
\text { Prior }\end{array} \\
\text { Performance, \% }\end{array}$ \\
\hline All multiligament knee injuries & 64.0 & $388.71 \pm 198.52$ & 50.0 & 30.0 \\
\hline ACL/MCL tears & 70.8 & $305.1 \pm 58.9$ & 68.2 & 43.5 \\
\hline ACL tear + PCL/LCL involvement & 55.6 & $459.2 \pm 245.1$ & 37.0 & 18.5 \\
\hline
\end{tabular}

$\mathrm{ACL}$, anterior cruciate ligament; LCL, lateral collateral ligament; MCL, medial collateral ligament; PCL, posterior cruciate ligament; RTP, return to play.

245.1 days for those with PCL/LCL involvement $(P=0.004)$ and $609.3 \pm 183.1$ days for those with frank dislocations $(P<$ $0.0001)$.

Of all athletes with multiligament knee injuries, 50.0\% were able to RTP during the season immediately after injury, and of these players, $76 \%$ were available in week 1 . A significantly greater proportion of players with ACL/MCL injuries (68.2\%) returned to play during the season immediately after injury compared with players with ACL and PCL/LCL involvement (37.0\%) $(P=0.047)$. Athletes with ACL/MCL injury were 3.85 times more likely to RTP during the season after injury compared with those with ACL and PCL/LCL involvement (odds ratio [OR], 3.85; 95\% CI, 1.01-14.80; $P=0.049)$. A higher proportion of players with $\mathrm{ACL} / \mathrm{MCL}$ injuries returned to play in a regular-season game the season after injury (68.2\% vs $26.7 \%$, $P=0.013)$. After RTP, both athletes with ACL/MCL tears and athletes with ACL and PCL/LCL involvement played in a similar number of possible games $(78.2 \% \pm 27.2 \%$ and $78.0 \% \pm 25.1 \%$, respectively). The effects of age $(P=0.67)$ and position $(P=$ $0.70)$ on RTP after a multiligament knee injury were evaluated, and neither was found to have a significant effect on RTP. Of all NFL athletes with multiligament knee injuries, 30.0\% were able to return to their prior level of performance; $43.5 \%$ of NFL athletes with ACL/MCL injuries returned to their prior level of performance; however, only $18.5 \%$ of NFL athletes with ACL and PCL/LCL involvement returned to their prior level of performance $(P<0.001)$. No players with knee dislocations were able to return to their prior level of performance $(P<$ 0.001) (Table 2).

\section{DISCUSSION}

RTP for NFL players with multiligament knee injuries is significantly less than the reported RTP rates for NFL players after isolated ACL tears. The current study noted that $64.0 \%$ of players returned to play after multiligament knee injuries, in contrast to significantly higher reported outcomes in the literature after isolated ACL tears in various professional sports. Reported rates of return for NFL players after isolated ACL tears range from $63 \%$ to $92 \%$, with the largest review demonstrating an RTP of $82.4 \%{ }^{2,5,8,13,14}$ Similar RTP rates are reported for isolated ACL tears in National Hockey League players. Sikka et $\mathrm{al}^{18}$ reported that $81 \%$ of hockey players were able to RTP for 1 or more seasons, with Erickson et $\mathrm{al}^{8}$ reporting an even higher RTP rate of $97 \%$. Furthermore, RTP in National Basketball Association players after isolated ACL tears has been reported to be between $78 \%$ and $84 \%{ }^{4,15}$

The current study demonstrates that RTP and performance strongly depends on specific characteristics of the multiligament knee injury. Players with ACL/MCL tears had significantly shorter time to RTP compared with athletes with ACL and PCL/LCL involvement or frank knee dislocation. Furthermore, a significantly greater proportion of players with ACL/MCL tears were able to return to their prior level of performance compared with athletes with ACL and PCL/LCL involvement. Players with ACL/MCL involvement also trended toward higher RTP rates compared with players with PCL/LCL involvement, although not statistically significant. These differences in outcomes between ACL/MCL injuries and ACL with PCL/LCL 
injuries may be related to their disparate injury mechanisms.

Furthermore, many MCL injuries heal nonoperatively, secondary to their intrinsic properties and healing environment. ${ }^{10-12,16}$ As a result, subsequent surgical intervention and outcomes often resemble an isolated ACL reconstruction.

The multidirectional and rotational forces resulting in multiligament knee injuries with PCL/LCL involvement can cause concomitant damage to other structures in the posterolateral corner as well as cartilaginous injury, resulting in continued rotational instability, pain and mechanical symptoms during athletic activity, and decreased performance postoperatively. ${ }^{6,19}$

The current study noted that only $30.0 \%$ of players with multiligament injuries returned to their prior level of performance. This is in contrast to RTP rates after isolated ACL tears in NFL athletes. Erickson et $\mathrm{al}^{8}$ and Carey et $\mathrm{al}^{5}$ demonstrated that $92 \%$ of NFL quarterbacks and $67 \%$ of NFL running backs/wide receivers returned to their preinjury level of performance after an isolated ACL tear. In addition, in a study of 98 NFL-caliber players with ACL reconstructions, Keller et $\mathrm{al}^{13}$ concluded that elite athletes can achieve equivalent levels of athletic performance compared with matched controls in drills such as the 40-yard dash, vertical leap, broad jump, shuttle run, and 3 -cone drill. Finally, Brophy et $\mathrm{al}^{3}$ demonstrated that isolated ACL reconstruction in NFL athletes did not affect a player's length of career in years or games played. The etiology of the relatively poor outcomes after multiligament knee injuries, compared with isolated ACL injury, is multifactorial. It is likely related to the more complex and high-energy traumatic mechanisms associated with multiligament knee injuries, longer/ more invasive surgical interventions, irreparable chondral damage, worse postoperative pain and symptoms, and prolonged postoperative immobilization with multiligament knee injuries.

Compared with other injuries in NFL athletes, this study shows that a multiligament knee injury can be particularly detrimental to a player's career. Mai et $\mathrm{al}^{14}$ conducted an extensive review of upper and lower extremity procedures in NFL players from 2003 to 2013. That study examined the RTP and performance of NFL players after ACL reconstruction, Achilles tendon repair, anterior cervical discectomy and fusion, lumbar discectomy, sports hernia repair, ankle fracture open reduction and internal fixation (ORIF), forearm ORIF, tibial intramedullary nail placement, and knee microfracture surgery. Only patellar tendon repair had a lower RTP rate than multiligament knee injuries. Furthermore, Mai et $\mathrm{al}^{14}$ reported that the majority of athletes with the noted injuries were able to return to their prior level of performance, which is not the case with multiligament knee injuries. This further demonstrates the devastating nature of a multiligament knee injury in an NFL athlete.

This study does have some limitations. First, it is a retrospective analysis, which introduces potential bias inherent to the study design. Players were treated at several institutions, by different surgeons, and using different surgical techniques. As a result, the types of grafts used for surgical reconstruction were not known and varied between athletes and could have confounded our results. Rehabilitation was also performed at different institutions, with no uniform rehabilitation protocol and under the guidance of different team physical therapists and athletic trainers. It is possible that variations in surgical technique and rehabilitation may have influenced a player's RTP. Second, our sample size, although comprehensive, was relatively small, with only 50 NFL players meeting our inclusion and exclusion criteria. In addition, this study does not account for other concomitant injuries that a player may have sustained, such as meniscal, patellar tendon, or cartilage injuries. Because injuries sustained as recently as the 2014 and 2015 seasons are included in the study, it is possible that some players may still RTP and continue to play more games. Therefore, our study may slightly underestimate RTP, number of games played, and performance. However, this limitation is mitigated by excluding players who sustained their injuries in the 2015 season who have not yet returned to play. Finally, the purpose of this study was to evaluate RTP at the same level of elite competition, and it does not account for players who may have returned to play in a different league or at a different level of competition.

Despite these limitations, this study provides clinically useful prognostic information regarding elite athletes with multiligament knee injuries. Though RTP after isolated ACL injuries has been well described for NFL players and other professional athletes, this is the first study to date to examine RTP, time to RTP, and performance levels of NFL players after multiligament knee injuries. These findings, collected from a comprehensive cohort of players at the highest level of competition, underscore the high morbidity associated with multiligament knee injuries and the challenges faced with returning to the same elite level of competition.

\section{CONCLUSION}

This study provides important prognostic information for NFL players sustaining multiligament knee injuries. The RTP rate for athletes with multiligament knee injuries is significantly less than the RTP rate for athletes with isolated ACL tears. In addition, athletes with ACL and MCL tears have a higher RTP rate, a significantly shorter time to RTP, and a greater likelihood of returning to prior performance than athletes with combined ACL and PCL/LCL tears.

\section{AUTHORS}

Neil K. Bakshi, MD (MedSport, Department of Orthopaedic Surgery, University of Michigan, Ann Arbor, Michigan); Moin Khan, MD (MedSport, Department of Orthopaedic Surgery, University of Michigan, Ann Arbor, Michigan); Simon Lee, MD (MedSport, Department of Orthopaedic Surgery, University of Michigan, Ann Arbor, Michigan); Fred T. Finney, MD

(MedSport, Department of Orthopaedic Surgery, University of Michigan, Ann Arbor, Michigan); Jeff Stotts, BS (Department of Athletic Training, St Vincent Health System, Little Rock, Arkansas); Robby S. Sikka, MD (TRIA Orthopaedic Center, 
Minneapolis, Minnesota and Mayo Clinic, Rochester, Minnesota); and Asheesh Bedi, MD (MedSport, Department of Orthopaedic Surgery, University of Michigan, Ann Arbor, Michigan).

\section{REFERENCES}

1. Boublik M, Schlegel T, Koonce R, Genuario J, Lind C, Hamming D. Patellar tendon ruptures in National Football League players. Am J Sports Med. 2011;39:2436-2440.

2. Bradley JP, Klimkiewicz JJ, Rytel MJ, Powell JW. Anterior cruciate ligament injuries in the National Football League: epidemiology and current treatment trends among team physicians. Arthroscopy. 2002;18:502-509.

3. Brophy RH, Gill CS, Lyman S, Barnes RP, Rodeo SA, Warren RF. Effect of anterior cruciate ligament reconstruction and meniscectomy on length of career in National Football League athletes: a case control study. Am J Sports Med. 2009;37:2102-2107.

4. Busfield BT, Kharrazi FD, Starkey C, Lombardo SJ, Seegmiller J. Performance outcomes of anterior cruciate ligament reconstruction in the National Basketball Association. Arthroscopy. 2009;25:825-830.

5. Carey JL, Huffman GR, Parekh SG, Sennett BJ. Outcomes of anterior cruciate ligament injuries to running backs and wide receivers in the National Football League. Am J Sports Med. 2006;34:1911-1917.

6. Coobs BR, LaPrade RF, Griffith CJ, Nelson BJ. Biomechanical analysis of an isolated fibular (lateral) collateral ligament reconstruction using an autogenous semitendinosus graft. Am J Sports Med. 2007;35:1521-1527.

7. Dwyer T, Marx RG, Whelan D. Outcomes of treatment of multiple ligament knee injuries. J Knee Surg. 2012;25:317-326.

8. Erickson BJ, Harris JD, Cole BJ, et al. Performance and return to sport after anterior cruciate ligament reconstruction in National Hockey League players. Orthop J Sports Med. 2014;2:2325967114548831.
9. Fanelli GC, Orcutt DR, Edson CJ. The multiple-ligament injured knee: evaluation, treatment, and results. Arthroscopy. 2005;21:471-486.

10. Hart RA, Woo SL, Newton PO. Ultrastructural morphometry of anterior cruciate and medial collateral ligaments: an experimental study in rabbits. J Orthop Res. 1992;10:96-103.

11. Indelicato PA, Hermansdorfer J, Huegel M. Nonoperative management of complete tears of the medial collateral ligament of the knee in intercollegiate football players. Clin Orthop Relat Res. 1990;256:174-177.

12. Johnson RJ, Beynnon BD, Nichols CE, Renstrom PA. The treatment of injuries of the anterior cruciate ligament. J Bone Joint Surg Am. 1992;74:140-151.

13. Keller RA, Mehran N, Austin W, Marshall NE, Bastin K, Moutzouros V. Athletic performance at the NFL Scouting Combine after anterior cruciate ligament reconstruction. Am J Sports Med. 2015;43:3022-3026.

14. Mai HT, Alvarez AP, Freshman RD, et al. The NFL Orthopaedic Surgery Outcomes Database (NO-SOD): the effect of common orthopaedic procedures on football careers. Am J Sports Med. 2016;44:2255-2262.

15. Minhas SV, Kester BS, Larkin KE, Hsu WK. The effect of an orthopaedic surgical procedure in the National Basketball Association. Am J Sports Med. 2016;44:10561061.

16. Nagineni CN, Amiel D, Green MH, Berchuck M, Akeson WH. Characterization of the intrinsic properties of the anterior cruciate and medial collateral ligament cells: an in vitro cell culture study. J Orthop Res. 1992;10:465-475.

17. Scranton PE Jr, Whitesel JP, Powell JW, et al. A review of selected noncontact anterior cruciate ligament injuries in the National Football League. Foot Ankle Int. 2997; 18:772-776.

18. Sikka R, Kurtenbach C, Steubs JT, Boyd JL, Nelson BJ. Anterior cruciate ligament injuries in professional hockey players. Am J Sports Med. 2016;44:378-383.

19. Sikka RS, Dhami R, Dunlay R, Boyd JL. Isolated fibular collateral ligamen injuries in athletes. Sports Med Arthrosc. 2015;23:17-21.

For reprints and permission queries, please visit SAGE's Web site at http://www.sagepub.com/journalsPermissions.nav. 